\title{
How to Perform and Interpret Upper Esophageal Sphincter Manometry
}

\author{
Shobna J Bhatia* and Chirag Shah \\ Department of Gastroenterology, Seth G S Medical College and K E M Hospital, Mumbai, India
}

Manometry of the pharynx and the upper esophageal sphincter (UES) provides important information on the swallowing mechanism, especially about details on the pharyngeal contraction and relaxation of the UES. However, UES manometry is challenging because of the radial asymmetry of the sphincter, and upward movement of the UES during swallowing. In addition, the rapidity of contraction of the pharyngoesophageal segment requires high frequency recording for capturing these changes in pressure; this is best done with the use of solid state transducers and high-resolution manometry. UES manometry is not required for routine patient care, when esophageal manometry is being performed. The major usefulness of UES manometry in clinical practice is in the evaluation of patients with oropharyngeal dysphagia.

(J Neurogastroenterol Motil 2013;19:99-103)

Key Words

Achalasia, cricopharyngeal; Dysphagia, oropharyngeal; Esophageal sphincter, upper; Manometry

\section{Introduction}

Esophageal manometry measures intraluminal pressures and coordination of pressure activity of the muscles of the esophagus, and is used to diagnose motility disorders of the esophagus. Performance of manometry requires a thorough understanding of the anatomy and physiology of the upper esophageal sphincter (UES) and pharynx, the manometric system, as also meticulous attention to technique. A poor-quality recording invariably leads to mistakes in interpretation. Esophageal manometry is currently the gold standard for assessment of esophageal motor activity. As far as the UES is concerned, manometry is complementary to radiology for the diagnosis of disorders. ${ }^{1} \mathrm{UES}$ disorders comprise of only $3 \%$ of manometry reports and are not included or described in major classifications of esophageal motility disorders (e.g., Chicago classification). ${ }^{2}$

\section{Special Considerations in Upper Esopha- geal Sphincter Manometry}

Challenges with UES manometry are both in performance and interpretation due to complex anatomy, asymmetry and movement of the sphincter. ${ }^{1}$ The UES has complex anatomy, and mainly comprises of inferior pharyngeal constrictor, cricopharyngeus and upper esophageal skeletal muscle, which is approximately 3-4 $\mathrm{cm}$ in length. ${ }^{3}$ The UES is asymmetric with the long axis being horizontally oriented. Due to radial asymmetry,

Received: December 25, 2012 Revised: None Accepted: December 29, 2012

(c) This is an Open Access article distributed under the terms of the Creative Commons Attribution Non-Commercial License (http://creativecommons. org/licenses/by-nc/3.0) which permits unrestricted non-commercial use, distribution, and reproduction in any medium, provided the original work is properly cited.

*Correspondence: Shobna J Bhatia, MD, DNB

Department of Gastroenterology, Seth G S Medical College and K E M Hospital, Acharya Dhonde Marg, Parel, Mumbai 400012 , India

Tel: +91-22-24136051 (extra. 7625),Fax: +91-22-24175626,E-mail: shobna.bhatia@gmail.com

Financial support: None.

Conflicts of interest: None. 
pressure values in the anteroposterior directions are considerably higher. The zone of highest pressure is located near the upper part of cricopharyngeus. Also, there is rapid upward movement of the sphincter during swallowing; this results in displacement of recording catheter during the procedure. ${ }^{3}$

UES manometry can be done with solid state high-resolution manometry or conventional water-perfused manometry system. Most manometry laboratories in the world now use high-resolution manometry, but conventional manometry continues to be used. Each system has own advantages and disadvantages (Table). ${ }^{4}$

All the muscles of the UES and pharynx are skeletal muscles, and have very rapid contraction with pressure rise rates up to 600 $\mathrm{mmHg} / \mathrm{sec}^{5}$; this means that the recording system should have a high frequency of capturing the changes in pressure, as is present in solid-state transducers. So far there has been no clinical need for high accuracy of pharyngeal pressure recording. Perfusion manometry can detect accurately the occurrence and onset of pharyngeal contraction and intrabolus pressure. However, water perfusion may cause cough during the procedure; an air-perfused side-hole sensor with perfusion rate of 2 to $8 \mathrm{~mL} / \mathrm{min}$ can be used to study the pharynx. ${ }^{6}$

The high-pressure zone of the UES is narrow and asymmetric; this requires multiple recording ports oriented radially, or circumferential sensors. Sleeve sensors can record basal UES pressure and the degree of UES relaxation with swallowing. However, the duration of UES relaxation recorded using these catheters is shorter, because the part of the sleeve located in the pharynx records the oncoming pharyngeal peristaltic wave, and the recording of UES relaxation is terminated. ${ }^{6}$ Due to move-

Table. Differences Between Water Perfused System and Solid State Catheters

\begin{tabular}{lc}
\hline \multicolumn{1}{c}{ Water-perfused point sensors } & \multicolumn{1}{c}{ Solid state catheters } \\
\hline Cheap and durable & $\begin{array}{c}\text { Extremely expensive and } \\
\text { company warranty for } \\
\text { few hundred procedures }\end{array}$ \\
$\begin{array}{l}\text { Inadequate frequency to } \\
\text { detect rapid changes }\end{array}$ & Adequate frequency response \\
$\begin{array}{l}\text { Water perfusate likely to induce } \\
\text { swallowing, cough and } \\
\text { possibly aspiration }\end{array}$ & No such disadvantage \\
$\begin{array}{l}\text { Incorporate multiple (up to 22) } \\
\text { recording points in order to provide } \\
\text { high-resolution pressure recordings }\end{array}$ & Because of high cost, multiple \\
Poor radial orientation & Good radial orientation if \\
& circumferential sensors used \\
\hline
\end{tabular}

ment of the UES during swallowing, the sensor is displaced from the high pressure zone. ${ }^{1}$ Therefore, the port that records the relaxation pressure should be at the upper edge of the UES.

For all these reasons, UES manometry is best done by a solid state catheter with built-in transducers; other options include a specially-designed sleeve sensor or an array of closely spaced side holes in water perfusion manometry. A recent study comparing manometry done with the water-perfused and solid state systems did not find significant differences in the values for UES resting pressure and length of sphincter. ${ }^{7}$

\section{How to Perform the Procedure}

\section{Equipment}

Procedure preparation requires a manometry laboratory setup. It should include procedure couch, chair, table and instrument setup including trolley and software.

At the beginning of procedure, proper functioning of the equipments should be verified. The recording catheter and its attachments should be checked. A calibration protocol should be run. ${ }^{8}$ In case of water-perfused system, the catheter should be inspected for leakage and blockage. Also, the flow of water should be checked, and air bubbles should be removed.

Make sure that ancillary equipments are available i.e., tape, gloves, lubricating jelly, local anesthetic, decongestant spray, cotton swabs, gauze pieces, bread or cookie pieces for solid swallow etc.

\section{Patient}

A detailed history should be taken, and indication for UES manometry be confirmed. All previous investigations (especially imaging and endoscopy) should be checked to assess whether structural lesions like strictures have been ruled out. Ask the patient for medication allergies, nasal deformities, and last food intake; 6-hour fasting prior to the procedure is adequate. The patient should be informed about the procedure. The patient should be instructed to avoid excessive swallowing. There should be no restrictive clothing around the neck. Patient's data should be entered in the database.

\section{Procedure}

\section{Intubation}

The patient may be in sitting or supine position; flexion of the neck helps to pass the catheter easily. Some clinicians like to anes- 
thetize the posterior pharynx with long cotton swab or spray. ${ }^{8}$ After application of local anesthetic/lubricating jelly, the catheter is gently placed parallel to the nasal floor and pushed to pharynx with feel of slight resistance at posterior pharyngeal wall. Slight resistance is felt at the level of UES; at this stage, the patient is asked to swallow saliva. In most cases, the catheter traverses the UES easily. If it does not, the patient can be given small sips of water using a straw. If this fails, the catheter should be introduced under endoscopic guidance. Once the catheter is placed in the esophagus, catheter position can be ascertained further by monitoring the display where a high pressure zone can be seen migrating up the screen. The most distal recording port should preferably be placed in the stomach, and fixed to the nose before recording starts.

\section{Data recording}

Manometric assessment of the pharynx and UES is ideally performed with the patient in sitting position. During the recording period, the patient's neck should be in the neutral position with no deflection to the side. Most centers prefer recording basal pressures for 2-5 minutes. After that, multiple swallows are giv$\mathrm{en}^{8}$ (10 swallows with $5 \mathrm{~mL}$ water and 10 swallows with $1 \mathrm{~mL}$ bread), and their response recorded. Further recording can be done if required (e.g., multiple rapid swallow, $200 \mathrm{~mL}$ water continuous swallow, air injection, or slow infusion of water or acid).

The role of a trained manometry technician/nurse cannot be under estimated. Uniformity in the procedure and a strictly followed protocol avoids unnecessary repeat procedures.

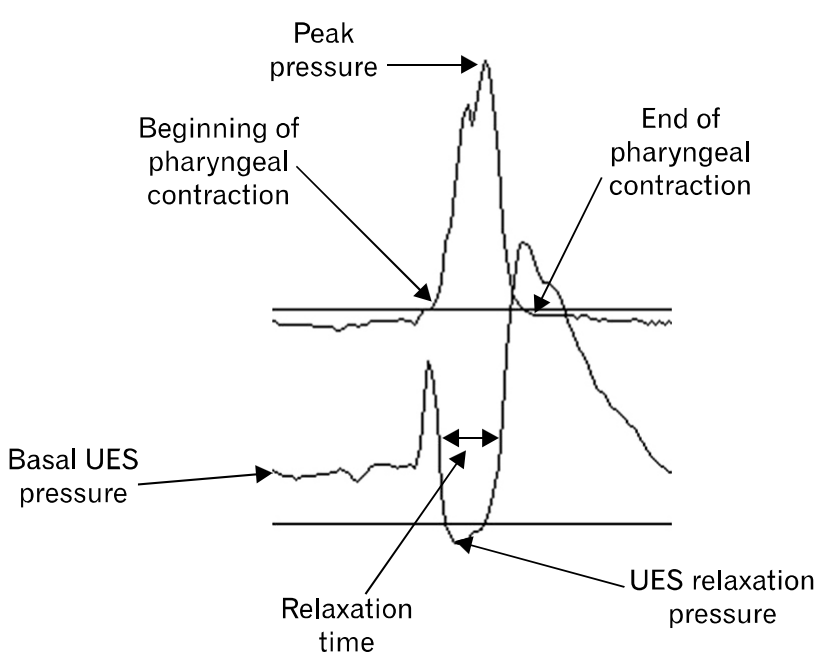

Figure 1. Line plot of upper esophageal sphincter and pharyngeal response to a swallow. UES, upper esophageal sphincter.

\section{Interpretation of Manometry}

All manometry systems that are currently available are computerized and have a dedicated software for capturing the images and analyzing data. The parameters which are most evaluated are baseline UES pressure, UES relaxation time, nadir pressure during UES relaxation, UES co-ordination with pharyngeal contraction, amplitude of pharyngeal contraction and intrabolus pressure. $^{3,9,10}$

\section{Baseline upper esophageal sphincter pressure}

There is no established normal range for UES pressure; previous studies have reported values between 100-150 mmHg. The values at the start of recording may be high due to catheter intolerance; hence it is better to measure UES pressure in the later part of the study and avoid repeated movement or repositioning of catheter during the procedure. ${ }^{6}$

UES pressure is affected by number of factors; the pressure is lower during sleep and anesthesia. Increased UES pressure may be recorded during emotional stress, speech, crying, with large bolus, increased downstream pressure and large catheter diameter. ${ }^{3}$ Decreased UES pressure may be recorded with lack of contact of catheter or pull of thyrohyoid muscle. ${ }^{6}$ Some factors that may alter values include flexibility, kinking and axial de-

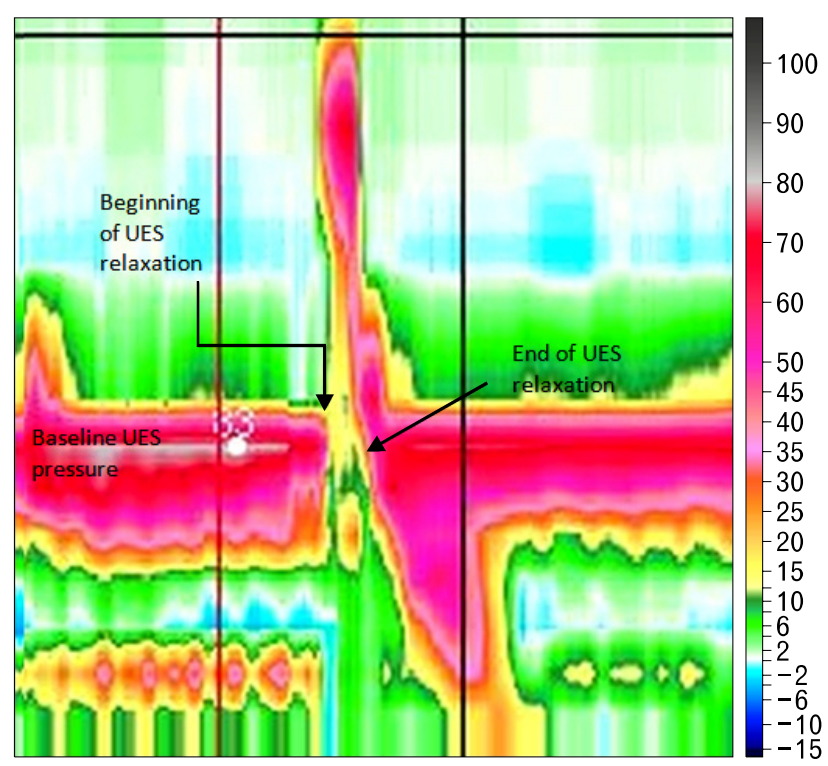

Figure 2. Spatiotemporal plots of water swallows from 1 subject. Catheter position is on the $y$-axis (position of upper esophageal sphincter [UES] is at $20 \mathrm{~cm}$ from the nares), time is on x-axis (time between vertical grid lines is $1 \mathrm{sec}$ ), and pressure is indicated by the color scale on the right of the figure. UES relaxation duration is time difference between start and end of relaxation. 
formity of the catheter, and blockage or air in the water channel. The significance of high baseline pressure is not known but may be associated with globus sensation in some patients. Intermittent high pressure may be seen with muscular disorders like myotonia. Data on UES pressure in normal subjects are sparse, and limited to subjects younger than 50 years.

\section{Upper esophageal sphincter relaxation}

The definition of UES relaxation is not standardized. UES relaxation requires inhibition of the cricopharyngeus and contraction of suprahyoid muscles. UES relaxation and opening are related, but not synonymous events. ${ }^{9}$ UES relaxation is a period of relatively stable low pressure when pressure falls to or below esophageal baseline pressure, and then rises above $50 \%$ of resting pressures (Fig. 1 and 2). ${ }^{1}$ It can also be calculated through complex computations and by automated analysis. But there is an element of subjectivity and difficulty in comparing studies because the start and end of relaxation are difficult to determine. Due to variability between individual swallows, it is not clear whether the final report should describe the average or range of UES relaxation pressures of all swallows, or the findings in a majority of swallows.

When measured with conventional manometry, UES relaxation period is 0.6 to 1.0 seconds; the range varies from $200-400$ milliseconds for dry swallow, and 300-500 milliseconds for $5 \mathrm{~mL}$ water swallows. ${ }^{1,5,9,10}$ The relaxation duration is longer when the bolus volume is increased ${ }^{10}$ as also for solid boluses. However, the average relaxation time measured by cricopharyngeus electromyography is 0.3 seconds. The difference is due to false impression of relaxation when UES moves upwards during a swallow; this error can be decreased with use of e-sleeve. ${ }^{8,10}$

Relaxation or residual pressure is the pressure recorded during relaxation of UES with respect to the esophageal pressure; it reflects nadir pressure during relaxation. During $5 \mathrm{~mL}$ water swallow, this pressure is usually below $15 \mathrm{mmHg}$. Ghosh et $\mathrm{al}^{10}$ reported that the relaxation pressure increased with increase in volume of the bolus and ranged from $8-13 \mathrm{mmHg}$ after a bolus of $20 \mathrm{~mL}$ was swallowed. High pressure usually indicates high downstream resistance. Dramatic increase can be seen with decrease in distensibility of pharyngoesophageal segments as in cases of cricopharyngeal bars and Zenker's diverticulum. Other causes of elevated nadir pressure include impaired relaxation of the UES, impaired anterior traction, large bolus swallow and extreme neck extension. ${ }^{9}$ It is important to note that failed UES relaxation is an observation, not a clinical diagnosis. Diminished UES opening can be a manifestation of disordered pharyngeal propulsion where the intrapharyngeal forces imparted by the advancing swallowed bolus are insufficient to open the sphincter to its fullest extent. The term UES achalasia means failure of relaxation of UES; unfortunately the term has incorrectly been used to describe a cricopharyngeal bar, and majority of reports on cricopharyngeal achalasia have not actually measured UES relaxation. ${ }^{9}$ Manometry or electromyography is necessary to detect failed UES relaxation, as the radiographic findings of a non-relaxing sphincter are nonspecific.'

Since the sequence of pharyngeal and cricopharyngeal motor events, including UES relaxation, originates in the medullary swallow center, it is not surprising that medullary lesions cause impaired UES relaxation; ${ }^{1}$ similar findings are also observed in Parkinson's disease and myopathy. ${ }^{11}$ In these cases, the abnormality may be detectable at manometry before the onset of dysphagia. This suggests that pharyngeal propulsion often compensates for UES non-relaxation, and that dysphagia severity is more related to the degree of associated pharyngeal dysfunction than failed UES relaxation alone.

\section{Contraction Pressures and Intrabolus Pre- ssure}

Hypopharyngeal intrabolus pressure is the low-pressure domain $(2-10 \mathrm{mmHg})$ within the bolus itself and is observed before the onset of the pressure upstroke caused by the advancing hypopharyngeal contraction. ${ }^{9,10}$ Intrabolus pressure varies according to the size and consistency of bolus, and resistance to bolus flow offered by the UES, and the major determinant of such resistance is sphincter diameter during deglutition. Ghosh et $\mathrm{al}^{10}$ developed an algorithm for calculating the relaxation interval and median intrabolus pressure. They defined median intrabolus pressure as the pressure at the 50 th percentile of the relaxation interval. They also defined a new parameter - the deglutitive sphincter resistance which was calculated as intrabolus pressure/relaxation interval.

\section{Upper Esophageal Sphincter Manometry in Disease States}

\section{Dysphagia}

Xue et $\mathrm{al}^{12}$ found that patients with dysphagia had higher prevalence of high UES residual pressure, weak pharyngeal contraction and UES/pharyngeal incoordination compared with patients presenting with other symptoms. Nearly $71 \%$ of dysphagia 
patients had at least one abnormality on their UES and pharyngeal manometry. However, they did not restrict the inclusion to oropharyngeal dysphagia.

Oculopharyngeal muscular dystrophy is associated with UES and pharyngeal incoordination, prolonged pharyngeal contraction, low pharyngeal pressure, and low pharyngeal contraction rate. ${ }^{13}$

Cricopharyngeal fibrosis and disordered neurally-mediated opening, and weak pharyngeal peristalsis cause incomplete opening of the UES. In Zenker's diverticulum, opening of the UES is impaired, and intrabolus pressure is increased. ${ }^{9}$ Identification of manometric abnormalities such as failed UES relaxation or elevated intrabolus pressure, may occasionally help decision-making related to cricopharyngeal myotomy or dilatation in carefully selected patients with oropharyngeal dysphagia ${ }^{1}$; in these cases, the response to dilatation or myotomy can be as high as $60-90 \% .^{14}$

\section{Globus sensation}

Globus is defined as a constant feeling of lump or fullness in the throat in absence of a structural lesion. Some studies have reported an association with a hypertensive UES. Data suggest that the symptom of globus may occur in two subsets of patients: female patients with a normal UES pressure, possibly having increased afferent sensation, and a group with equal sex distribution but abnormally elevated UES resting pressure. ${ }^{15}$

\section{Conclusion}

UES manometry is useful in the management of patients with oropharyngeal dysphagia. It is a technically demanding procedure due to the anatomy of the UES, as is the movement of the sphincter during swallowing.

\section{References}

1. Hila A, Castell JA, Castell DO. Pharyngeal and upper esophageal sphincter manometry in the evaluation of dysphagia. J Clin Gastroenterol 2001;33:355-361.
2. Wang YT, Yazaki E, Sifrim D. High-resolution manometry: esophageal disorders not addressed by the "Chicago classification". J Neurogastroenterol Motil 2012;18:365-372.

3. Singh S, Hamdy S. The upper oesophageal sphincter. Neurogastroenterol Motil 2005;17(suppl 1):3-12.

4. Pandolfino JE, Kahrilas PJ, American Gastroenterological Association. AGA technical review on the clinical use of esophageal manometry. Gastroenterology 2005;128:209-224.

5. Cook IJ, Kahrilas PJ. American Gastroenterological Association technical review on management of oropharyngeal dysphagia. Gastroenterology 1999;116:455-478.

6. Holloway RH. Esophageal manometry. GI Motility online 2006. doi:10.1038. Available from URL: http://www.nature.com/gimo/ contents/pt1/full/gimo30.html (accessed 31 Dec 2012).

7. Wang K, Duan LP, Ge Y, Xia ZW, Xu ZJ. A comparative study of 22-channel water-perfusion system and solid-state system with 36-sensors in esophageal manometery. BMC Gastroenterol 2012;12:157.

8. Shaker R, Hofmann C. How to perform esophageal manometry. GI Motility online 2006. doi:10.1038. Available from URL: http://www. nature.com/gimo/contents/pt1/full/gimo90.html (accessed 31 Dec 2012).

9. Cook IJ. Clinical disorders of the upper esophageal sphincter. GI Motility online 2006. Available from URL: http://www.nature.com/gimo/contents/pt1/full/gimo37.html (accessed 31 Dec 2012).

10. Ghosh SK, Pandolfino JE, Zhang Q, Jarosz A, Kahrilas PJ. Deglutitive upper esophageal sphincter relaxation: a study of $75 \mathrm{vol}-$ unteer subjects using solid-state high-resolution manometry. Am J Physiol Gastrointest Liver Physiol 2006;291:G525-G531.

11. Ali GN, Wallace KL, Schwartz R, de Carle DJ, Zagami A, Cook IJ. Mechanisms of oral-pharyngeal dysphagia in patients with Parkinson's disease. Gastroenterology 1996;110:383-392.

12. Xue S, Katz PO, Castell JA, et al. Upper esophageal sphincter and pharyngeal manometry: which patients? [abstract]. Gastroenterology 2000;118:A410

13. Castell JA, Castell DO, Duranceau C, Topart P. Manometric characteristics of the pharynx, upper esophageal sphincter, esophagus, and lower esophageal sphincter in patients with oculopharyngeal muscular dystrophy. Dysphagia 1995;10:22-26.

14. Hatlebakk JG, Castell JA, Spiegel J, Paoletti V, Katz PO, Castell DO. Dilatation therapy for dysphagia inpatients with upper esophageal sphincter dysfunction: manometric and symptomatic response. Dis Esophagus 1998;11:254-259.

15. Corso MJ, Pursnani KG, Mohiuddin MA, et al. Globus sensation is associated with hypertensive upper esophageal sphincter but not with gastroesophageal reflux. Dig Dis Sci 1998;43:1513-1517. 